\title{
La tarea de seguimiento en el estudio de la atención selectiva
}

\author{
JuAn José Ortells Rodríguez \\ Luis José Fuentes Melero
}

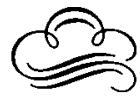

\section{Resumen}

La utilización de la tarea de seguimiento en el estudio de la atención selectiva, ba dado lugar a un gran número de resultados confusos y contradictorios que ban influido en el mantenimiento de la controversia sobre el problema de la selectividad.

El objetivo del presente articulo fue analizar algunos factores responsables de esta discrepancia experimental:

1. Problemas metodológicos asociados con los estudios de seguimiento.

2. Variables relacionadas con la tarea de seguimiento cuya influencia no ba sido tomada en cuenta en la literatura.

3. Concepciones teóricas extremas sobre la atención selectiva.

El análisis realizado sugiere un replanteamiento de los problemas de selectividad y capacidad, donde se cuestione la dicotomía selección anterior/posterior y en su lugar se asuman concepciones más flexibles del proceso atencional.

\section{Abstract}

The numerous equivocal results of the studies using a shadowing task in the investigation of the selective process, have favoured the maintenance of the controversy about the selectivity problem.

The purpose of the present article was to analyse some factors responsibles of this experimental discrepancy:

framework of facticsciences, the properties that exclusively belong to 1. Methodological problems associated with the shadowing studies.

2. Some variables related with the shadowing task whose influence bas not been considered in literature.

3. Extremes theoretical conceptions about the selective attention.

The analysis that we bave realized suggest a new survey of the selectivity and capacity problems, where it is quetionned the early/late selection dicotomy and in this place it is assuming more flexibles conceptions about the attentional process. 
El estudio de la atención selectiva ha sido objeto de una extensa investigación experimental, en la que se han desarrollado tareas y técnicas muy diversas. Entre ellas destaca la de seguimiento o «sbadowing" por ser una de las más utilizadas en el estudio del proceso selectivo y que ha dado lugar sin embargo a un mayor número de resultados contradictorios.

Esta técnica consiste en presentarle a los sujetos dos mensajes verbales en forma dicótica o binaural, instruyéndoles para que repitan en voz alta palabra por palabra, uno de los dos mensajes a medida que lo oyen.

El prime autor que utilizó el seguimiento fue Cherry (1953), con el objeto de averiguar el nivel de procesamiento que alcanzan los estímulos presentados por un canal no atendido. Este autor pensó que el shadowing constituiría un buen método para controlar la atención y garantizar que el mensaje simultáneo fuera verdaderamente ignorado. Por una parte, el tener que repetir continuamente un mensaje absorvería toda la atención del individuo debido a la dificultad que representa dicha tarea y al grado de concentración que se requiere. Además, podría controlarse la ocurrencia de errores ocasionales en la realización del seguimiento, lo que sugeriría la posibilidad de cambios de atención hacia el mensaje secundario que debía ignorarse.

El trabajo de Cherry (1953) demostró que los sujetos sólo captaban diferencias en las características físicas de los mensajes no atendidos, como el cámbio de voz de masculina a femenina, pero en ningún caso detectaban las variaciones producidas en el significado de dicho mensaje.

Los resultados de algunos estudios posteriores (v.g. Moray, 1959) demostraron sin embargo, que es posible advertir algunos estímulos no atendidos de especial relevancia, como el propio nombre del sujeto.

Estos resultados dieron lugar al desarrollo de una importante controversia teórica, en particular entre dos tipos de modelos alternativos conocidos como teorías de selección temprana (v.g. Broadbent, 1958, 1971; Treisman, 1960, 1964) y teorías de selección tardía (v.g. Deutsch y deutsch, 1963; Norman, 1968).

Ambos modelos coinciden en asumir que el cerebro es limitado en su capacidad para procesar información, de ahí la necesidad de un proceso selectivo. Las discrepancias radican esencialmente en la localización de dicho mecanismo a lo largo de las distintas etapas de procesamiento.

Para el primer tipo de teorías (early selection) la etapa en que opera la selección ocurre antes de la percepción de los estímulos (Broadbent, 1958) o durante la misma (Broadbent, 1971; Treisman, 1960), de modo que sólo la información atendida recibirá un procesamiento ulterior a nivel semántico. Sin embargo, para las teorías de selección tardía (late selection) la etapa en la que opera dicho mecanismo ocurre después de la percepción de los estímulos, bien en la memoria (Norman, 1968) o durante la organización y selección de las respuestas (Deutsch y Deutsch, 1963), de modo que toda la información, atendida o no, se procesaría a nivel del significado.

La investigación posterior trató principalmente de aportar nueva evidencia en favor de un análisis semántico de la información no atendida (v.g. Corteen y Wood, 1972; Lewis, 1970; Smith y Groen, 1974; Von Wright, Anderson y Stenman, 1975) o en mostrar una ausencia del mismo (v.g. Davis y Smith, 1972; Treisman y Geffen, 1967; Treisman, Squire y Green, 1974; Wardlaw y Kroll, 1976). Gran parte de estos estudios llegaron a resultados opuestos utilizando procedimientos experimentales muy similares (v.g. Glucksberg y Cowen, 1970 y Norman, 1969; Lewis, 1970 y Treisman, Squire y Green, 1974). 
El objetivo del presente artículo consiste en analizar los distintos factores que pueden haber influído en el mantenimiento de esta controversia sobre el problema de la selectividad.

\section{POSIBLES CAUSAS DE LAS DISCREPANCIAS EXPERIMENTALES}

\section{Dificultades metodológicas}

Es probable que gran parte de la discrepancia experimental obtenida, pueda explicarse por la existencia de algunas inadecuaciones metodológicas entre las que destacamos las siguientes:

\section{Situaciones contradictorias}

Algunos estudios previos se han caracterizado por colocar al sujeto en una situación de «doble ciego» (Underwood, 1976). Por una parte, se instruía a los sujetos para que siguieran los estímulos presentados por el canal atendido y por otra, se les pedía que respondieran ocasionalmente a determinados items del canal no atendido. El hecho de encontrar un porcentaje de respuestas muy bajo ante los estímulos supuestamente ignorados, ha sido interpretado por algunos autores (v.g. Treisman y Geffen, 1967) como un problema perceptual.

Sin embargo, es posible una interpretación alternativa, en el sentido de que responder continuamente a un mensaje como requiere el seguimiento, impida cualquier respuesta simultánea a los estímulos no atendidos.

\section{Instrucciones inadecuadas}

Con frecuencia, las instrucciones empleadas en lo que se refiere a concentrar o dividir la atención en una tarea de audición dicótica, han sido confusas. Así, se pedía a los sujetos que ignorasen un mensaje secundario y a la vez se les preguntaba por ciertos aspectos del mismo a lo largo de la sección experimental (v.g. Norman, 1969). En tales circunstancias, es poco probable que dicha información fuera realmente ignorada, pues como señalan algunos autores (v.g. Davis y Smith, 1972; Lyons, 1974), estas instrucciones incrementarían la «importancia» del mensaje no atendido, pudiendo provocar cambios de atención sin que lo pretenda el experimentador.

Esto sugiere la necesidad de que las instrucciones pongan de manifiesto el carácter distractor del mensaje secundario para garantizar que sea realmente ignorado (v.g. Fuentes y Tudela, 1982; Ortells y Tudela, en prensa).

\section{Medidas de recuerdo poco fiables}

Los primeros estudios de seguimiento se caracterizaron por tomar como índice de procesamiento no atendido las respuestas verbales de los sujetos sobre dicho mensaje, una vez finalizada la sesión experimental.

La ausencia de respuestas acerca del significado de la información no atendida, ha sido interpretada generalmente como evidencia de que los estímulos que llegan al organismo no son procesados a nivel superior (v.g. semántico) a menos que reciban el beneficio de la atención consciente. Sin embargo, este argumento es cuestionable por varios razones:

Por una parte, en la mayoría de estos estudios no se controlaba el intervalo de tiempo transcurrido entre la presentación del material sobre el que se iba 
a preguntar y el test de recuerdo (v.g. Broadbent y Gregory, 1963; Cherry, 1953; Moray, 1959). Una demora considerable podría hacer que desapareciera el trazo de memoria de las palabras no atendidas, en el supuesto de que hubieran sido percibidas.

En segundo lugar, por los resultados de algunos estudios que demostraron la ocurrencia de procesamiento semántico no atendido, utilizando otras variables dependientes (como alternativa a la respuesta verbal), tales como el tiempo de reacción (v.g. Lewis, 1970) o una respuesta no verbal (v.g. psicogalvánica) ante los estímulos no atendidos (v.g. Corteen y Wood, 1972).

Esto demuestra que el hecho de preguntarle al sujeto si recuerda algo de un mensaje previamente ignorado, es una medida poco sensible para detectar indicios de codificación no atendida.

En aquellos casos en los que se trata de determinar únicamente si un tipo de información está o no presente en la memoria, sería más apropiado emplear una medida alternativa al recuerdo, como es el reconocimiento, cuya eficacia ha sido probada en distintas investigacones (v.g. Dawson y Reardon, 1973; Fuentes y Tudela, 1982; Ortells, 1982).

Sin embargo en algunos estudios, la utilización de pruebas de reconocimiento no ha sido suficientemente fiable, encontrándose ciertos fallos metodológicos:

- Cuando el test de reconocimiento está constituido por items muy similares (v.g. Norman, 1969), una puntuación baja en el mismo podría suponer un problema discriminativo y no de memoria (Kellogg, 1980).

- La ejecución de los sujetos en dicha prueba, se ha medido con frecuencia utilizando el porcentaje de respuestas correctas o de aciertos y falsas alarmas (v.g. Kellogg, 1980). La aplicación de la teoría de detección de señales a las tareas de reconocimiento, nos proporciona un índice de discriminabilidad de los sujetos, no «contaminado» por posibles sesgos en sus respuestas (v.g. Fuentes y Tudela, 1982; Ortells y Tudela, en prensa).

\section{Variables relacionadas con la tarea de seguimiento}

El seguimiento es una tarea compleja que permite la manipulación y control de una serie de variables relacionadas con la misma. Es probable que gran parte de la evidencia experimental contradictoria en los estudios que utilizaron dicha tarea, se deba a la influencia de algunas de estas variables, entre las que destacan las siguientes:

\section{Tipo de mensaje seguido}

Es posible que el nivel de procesamiento alcanzado sobre un canal no atendido (semántico y/o fonético) varíe en función del tipo de «material» utilizado para la realización del seguimiento: prosa, palabras sin relación entre sí o palabras sin sentido.

Así, el mensaje en prosa se caracteriza entre otras cosas por la repetición de una serie de frases o palabras (v.g. sustantivos, adjetivos) que no añaden información nueva a su significado. A esto se le llama «redundancia».

En tales circunstancias, el sujeto puede seguir dicho mensaje sin necesidad de que se concentre plenamente, lo que podría facilitar el procesamiento del canal no atendido. No ocurriría igual cuando se emplean otros tipos de material, como palabras no relacionadas o sin sentido. En estos casos, la realización adecuada del seguimiento requeriría una mayor concentración del sujeto. 


\section{9}

Sullivan (1976) demostró que al aumentar la redundancia del mensaje «seguido», ocurría un incremento proporcional en la detección de items presentados por el otro canal.

De esta forma, es posible que el seguimiento de uno u otro tipo de «material» implique situaciones cualitativamente diferentes, pudiendo ésto influir en la obtención de una evidencia experimental contradictoria.

\section{Tasa de presentación}

Aunque se ha demostrado la importancia de esta variable por sí sola, no sucede así con respecto a su posible interacción con otras variables. De esta forma, el efecto de la tasa de presentación de los items seguidos, puede depender del tipo de material que se utilice así como de la mayor o menor práctica que tenga el sujeto con la tarea.

\section{Práctica con la tarea}

Debido a la dificultad que supone el seguimiento, los sujetos son generalmente sometidos a una serie de ensayos de práctica, para que consigan familiarizarse con dicha tarea y logren una ejecución estable con pocos o ningún error. En estos casos, la práctica se ha utilizado como un procedimiento de control, sin estudiarse su posible influencia en el procesamiento de la información no atendida.

Por otra parte, los estudios que han manipulado la práctica con el seguimiento, utilizaron niveles muy altos de dicha variable (v.g. Underwood, 1974; Underwood y Moray, 1971) para comprobar si con ello se facilitaba la realización de varias tareas simultáneas, en una situación de atención dividida.

Sin embargo, en algunos trabajos recientes que emplearon niveles de práctica inferiores (v.g. Ortells, 1982), se demostró su influencia en el sentido de facilitar el procesamiento semántico de estímulos no atendidos, más bien que inhibirlo, como asumían algunos autores (v.g. Treisman, Squire y Green, 1974). Estos resultados ponen de manifiesto la importancia de dicha variable, aun cuando se utilice como una forma de control, pues es posible que en función del tipo de material utilizado y/o de la tasa de presentación de las palabras, se requieran diferentes ensayos de práctica para conseguir una ejecución estable en la tarea.

\section{Frecuencia de las palabras no atendidas}

Numerosos estudios han demostrado que las palabras de baja frecuencia de un mensaje atendido, se teconocen mucho mejor que las de alta frecuencia (v.g. Shepard, 1967; Underwood y Freund, 1970).

Sin embargo, pocos autores han investigado el efecto de esta variable en la detección de estímulos no atendidos. En un trabajo reciente (Ortells, 1982), se demostró que las palabras de baja frecuencia no atendidas eran mejor reconocidas que las de alta frecuencia, en particular, cuando los sujetos tenían mucha práctica en el seguimiento de un mensaje simultáneo.

Este hecho podría explicar la controversia obtenida en algunos estudios previos que no manipularon dicha variable. Es probable que en función del tipo de frecuencia que predominara, se encontrara o no evidencia de procesamiento semántico no atendido. 


\section{0}

Oido de presentación

Dawson y Schell (1982), utilizando la respuesta psicogalvánica como variable dependiente, demostraron que la detección del significado de estímulos no atendidos, sólo era posible cuando estos se presentaban por el oído izquierdo.

Para Dawson y Schell, este resultado podía aclarar la discrepancia experimental de algunas investigaciones que habían utilizado un procedimiento similar. Así, en los estudios que presentaban el mensaje no seguido por el oído izquierdo, se demostró que los sujetos detectaban cambios en su significado (v.g. Corteen y Dunn, 1974; Corteen y Wood, 1972; Forster y Govier, 1978). Sin embargo, en aquellos estudios que contrabalancearon el oído de presentación, no se encontró evidencia de codificación semántica no atendida (v.g. Wardlaw y Kroll, 1976).

\section{CONSIDERACIONES TEORICAS}

Independientemente de los problemas metodológicos ya mencionados, el mantenimiento de la controversia se ve favorecido por la existencia de algunas posiciones teóricas extremas sobre la localización y funcionamiento del mecanismo selectivo así como de la capacidad de procesamiento.

\section{Concepciones teóricas opuestas sobre la atención selectiva}

Tanto los modelos de selección temprana como los de selección tardía, asumen un solo lugar de selectividad como el más importante. Sin embargo, «admitir» información en la conciencia es concebido como "percepción» por los primeros y como «respuesta» por los segundos. Esto da lugar, como señala Underwood (1976) a que un mismo resultado se interprete de modo distinto por ambas teorías. Tal es el caso de los resultados obtenidos por Cherry (1953), que para Broadbent (1958) constituían una evidencia en favor de su modelo de selección temprana, mientras que Deutsch y Deutsch (1963) los interpretaron como datos en apoyo de un modelo de selección tardía o post-perceptual.

Es probable que una postura intermedia entre estas concepciones extremas, sea más coherente con la realidad y puede explicar adecuadamente los diversos resultados que aparecen en la literatura sobre la atención selectiva.

Broadbent (1971) realizó una modificación de su modelo de 1958 que le llevó a sugerir una importante distinción entre dos tipos de atención selectiva: «stimulus set» y «response set». El primero aparecería cuando son las características físicas las que diferencian los estímulos relevantes e irrelevantes y por tanto la selección ocurriría en una etapa anterior a la percepción. El segundo tipo actuaría cuando las características diferenciadoras están en el conjunto de respuestas diferentes a cada clase de estímulos. El modo de selección «response set» dependería del contacto del input sensorial con la información almacenada en la memoria. Esta distinción ha sido apoyada posteriormente (v.g. Keren, 1976).

Otros autores postulan una teoría multimodal de la atención, donde tendrían cabida todos los modelos de selección (v.g. Johnston y Heinz, 1978). En este sentido, serán las características de los estímulos y las demandas de las tareas las que determinarán la actuación o relevancia de uno u otro tipo de atención selectiva. 


\section{1}

\section{El problema de la capacidad de procesamiento}

Los modelos tradicionales sobre la atención selectiva han considerado al organismo como un sistema de capacidad limitada. La idea dominante es que se trata de una limitación estructural fija, debido al soporte fisiológico que subyace al proceso. Para este tipo de teorías (que asumen un canal único de procesamiento) sólo podrán realizarse dos tareas simultáneamente si se cambia rápidamente la atención de un canal a otro. Sin embargo, la validez y alcance de este supuesto ha sido cuestionado desde diferentes perspectivas:

- Algunos autores han propuesto la existencia de múltiples canales de procesamiento, de modo que los sujetos serían capaces de realizar dos tareas al mismo tiempo si ambas utilizan «subsistemas» de procesamiento distintos (v.g. Allport, 1980; Allport, Antonis y Reynolds, 1972).

- Para otros autores, el organismo dispone de unos «recursos de procesamiento», de forma que la limitación de capacidad no se localiza en ninguna estructura específica, sino que es más bien general (v.g. Kahneman, 1973). Si una tarea demanda poca cantidad de recursos, aún quedarían disponibles para realizar una segunda tarea, siempre que éstos no sobrepasen la cantidad total de recursos disponibles en el sistema. Si fuese así, se produciría una interferencia entre ambas tareas.

En palabras de Norman y Bobrow (1975), es posible que la tarea de seguimiento esté limitada por los «datos» (características implícitas a la tarea) y no agote por tanto la cantidad de recursos de que dispone el organismo. De esta forma, aún quedarían recursos para poder identificar el material presentado por el canal no atendido sin tener que recurrir al cambio de atención. (Fuentes, 1981).

- Por último, existe una amplia evidencia experimental (v.g. LaBerge, 1973; Schneider y Shiffrin, 1977; Shiffrin y Schneider, 1977) más consistente con la idea de asumir cierta «flexibilidad» en los límites de capacidad, pudiendo ésta variar en función de determinados factores, tales como el aprendizaje. Así, el funcionamiento de la atención podría estudiarse como una habilidad.

Todo esto ha dado lugar a que últimamente gran parte del interés en el proceso atencional se haya concentrado en los aspectos relativos a su capacidad. Sin lugar a dudas, un conocimiento más exhaustivo del problema de la capacidad de procesamiento relacionada con la tarea de seguimiento, obligará a replantear las concepciones existentes sobre la atención selectiva y la importancia de dicha tarea en su estudio.

El análisis realizado en este artículo sugiere la inadecuación de las teorías que asumen una concepción dicotómica sobre el proceso selectivo así como de los estudios que han tenido únicamente por objeto el aportar evidencia en favor o en contra de dichos modelos. Este trabajo sugiere la necesidad de que la investigación futura tenga como objetivo primordial el análisis minucioso de las características implícitas a las tareas utilizadas (v.g. el seguimiento) y el de las distintas variables que pueden afectar al procesamiento de la información. 


\section{Referencias}

Allport, D. A. (1980): Attention and Perfomance. En G. Claxton (ed.): Cognitive Psychology; New directions. Londres, Rontledge and K. Paul.

Allport, D. A., Antonis, B. y Reynolds, P. (1972): On the division of attention: A disproof of the single channel hypothesis. Quarterly Journal of Experimental Psychology, 24, 225-235.

Broadbent, D. E. (1958): Perception and communication. Nueva York: Pergamon Press.

Broadbent, D. E. (1971): Decision and stress. Nueva York: Academic Press.

Broadbent, D. E. y GreGory, M. (1963): Division of attention and the decision theory of singnal detection. Proceedings of the Royal Society, 158, 222-232.

CorteEn, R. S. y Dunn, D. (1974): Shock-associated words in a nonattended message: A test for momentary awareness. Journal of Experimental Psycbology, 102, 1.143-1.144.

CorteEn, R. S. y WoOD, B. (1972): Autonomic responses to shock-associated words in an unattended channel. Joumal of Experimental Psychology, 94, 308-313.

CherRY, E. C. (1953): Some experiments on the recognition of sppech with one and with two ears. Journal of the Acoustical Society of America, 25, 975-979.

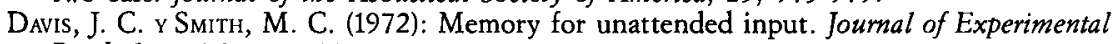
Psychology, 96, 380-388.

Dawson, M. E. y REARdON, P. (1973): Construct validity of recall and recognition postconditioning measures of awaeness. Joumal of Experimental Psychology, 98, 308-315.

Dawson, M. E. y SCHELL, A. M. (1982): Electrodermal responses to attended and nonattended significant stimuli during dichotic listening. Joumal of Experimental Psychology: $\mathrm{Hu}$ man Perception and Perfomance, 8, 315-324.

Deutsch, J. A. y Deutsch, D. (1963): Attention: some theorectical considerations. Psychological Review, 70, 80-90.

ForsTER, P. M. Y GoviER, E. (1978): Discrimination without awareness. Quarterly Journal of Experimental Psychology, 30, 289-295.

FUentes, L. J. (1981): Memoria a corto y largo plazo para los items atentidos y no atendidos. Memoria de Licenciatura. Univ. de Granada.

FUENTES, L. J. Y TUDELA, P. (1982): Memoria a corto y largo plazo para la información atendida y no atendida. Revista de Psicologia General y Aplicada, 37, 675-695.

Johnston, W. A. y HeINZ, S. P. (1978): Flexibility and capacity demands of attention. Journal of Experimental Psychology: General, 107, 420-435.

Kahneman, D. (1973): Attention and effort. Englewood Clifs, N. J.: Prentice Hall.

KeLLoG, R. T. (1980); Is conscious attention necesary for long-term storage? Journal of Experimental Psychology: Human Learning and Memory, 16, 379-390.

KEREN, G. (1976): Some considerations of two alleged kinds of selective attention. Journal of Experimental Psychology: General, 105, 349-374.

Laberge, D. (1973): Attention and the measurement of perceptual learning. Memory and Cognition, 1, 268-276.

LEwIS, J. L. (1970): Semantic processing of unattended messages using dichotic listening. Journal of Experimental Psychology, 85, 225-228.

LYONS, J. L. (1974): The encoding of ignored information. Memory and Cognition, 2, 161-168.

MoRAY, N. (1959): Attention in dichotic listening: affective cues and the influence of instructions. Quarterly Journal of Experimental Psychology, 11, 56-60.

Norman, D. A. (1968): Toward a theory of memory and attention. Psychological Review, 75 , 522.536 .

Norman, D. A. (1969): Memory while shadowing. Quarterly Joumal of Experimental Psycho. $\log y, 21,85-93$.

Norman, D. A. y Bobrow, D. G. (1975): On data-limited and resource-limited processes. Cognitive Psychology, 7, 44, 64.

OrTelLs, J. J. (1982). Efecto de la cantidad de práctica y de la frecuencia de las palabras en la detección no atendida. Tesis de Licenciatura presentada en el Departamento de Psicología de la Universidad de Granada.

Ortells, J. J. y Tudela, P. (en prensa). Efecto del nivel de práctica y de la frecuencia de las palabras, en el procesamiento de la información no atendida.

SCHNEIDER, W. y SHIFFrIN, R. M. (1977): Controlled and automatic human information processing: I. Detection, Search and Attention. Psychological Review, 84, 1-66.

SHEPARD, R. N. (1967): Recognition memory for words, sentences and pictures. Journal of Verbal Leaming and Verbal Bebavior, 6, 156-163.

SHIfFRIN, R. M. y SCHNeIDER, W. (1977): Controlled and automatic human information processing: II. Perceptual Learning, Automatic Attending and a General Theory. Psychological Review, 84, 127-186. 
Smith, M. C. y Groen, M. (1974): Evidence for semantic analysis of unattended verbal items. Joumal of Experimental Psychology, 102, 595-603.

Sullivan, L. (1976): Selective attention and secondary message analysis: A reconsideration of Broadbent's filter model of selective attention. Quarterly Journal of Experimental Psychology, 28, 167-178

Treisman, A. M. (1960): Contextual cues in selective listening. Quarterly Journal of Experimental Psychology, 12, 242-248.

Treisman, A. M. (1964): Effect of irrelevant material on the efficiency of selective listening. American Joumal of Psycbology, 77, 533-546.

Treisman, A. M. y Geffen, G. (1967): Selective attention: Perception or response? Quarterly Journal of Experimental Psychology, 19, 1-17.

Treisman, A. M.; Souire, R. y Green, J. (1974): Semantic processing in dichotic listening? A replication. Memory and Cognition, 2, 641-646.

UNDERWOOD, G. (1974): Moray vs. the rest: The effect of extended shadowing practice. Quarterly Journal of Experimental Psychology, 26, 368-372.

UNDERWOOD, G. (1976): Semantic interference from unattended printed words. British Journal of Psychology, 67, 327-338.

UNDERWoOd, B. J. Y FreUnd, J. S. (1970): Word frequency and short-term recognition memory. American Joumal of Psychology, 83, 343-351.

UNDERWOOD, G. y MORAY, N. (1971): Shadowing and monitoring for selective attention. Quarterly Journal of Experimental Psychology, 23, 284-295.

VON W RIGHT, J. M.; Anderson, K. y Stenman, V. (1975): Generalization of conditioned GSRs in dichotic listening. In P.M.A. Rabbitt and S. Dornic (Eds.), Attention and Perfomance, vol. IV. Nueva York: Academic Press.

W ARDLAW, K. A. y Kroll, N. E. A.: (1976): Autonomic responses to shock-associated in a nonattended message: A failure to replicate. Joumal of Experimental Psychology: Human Perception and Performance, 2, 357-360. 